Tropical Journal of Pharmaceutical Research March 2019; 18 (3): 491-497

ISSN: $1596-5996$ (print); 1596-9827 (electronic)

(C) Pharmacotherapy Group, Faculty of Pharmacy, University of Benin, Benin City, 300001 Nigeria.

Available online at http://www.tjpr.org

Original Research Article

http://dx.doi.org/10.4314/tjpr.v18i3.7

\title{
MicroRNA-124 regulates apoptosis in sevoflurane anesthesia-induced neuroblastoma cells by targeting enhancer of zeste homolog 2
}

\author{
Lingyun Wei, Jiansheng Fang, Yiqi Fang, Chengnv Li \\ Department of Anesthesiology, Chunan First People's Hospital, Chunan, Zhejiang, 311700, China
}

*For correspondence: Email: lichengnvll@163.com; Tel: +86-571-65025815

Sent for review: 17 November 2018

Revised accepted: 25 February 2019

\begin{abstract}
Purpose: To investigate the mechanism of microRNA-124 action on neuroblastoma apoptosis induced by sevoflurane.

Methods: MiR-124 expression was assessed in a neuroblastoma cell line (SMS-KAN) using quantitative reverse transcription polymerase chain reaction (qRT-PCR). The role of miR-124 in sevoflurane anesthesia-induced neuroblastoma was studied by cell activity and apoptosis analysis using 3-(4, 5-dimethylthiazolyl-2-yl)-2-5 diphenyl tetrazolium bromide (MTT) assay and flow cytometry, respectively. MiR-124 target protein genes were confirmed via luciferase reporter activity, qRT-PCR, and western blot analysis.

Results: miR-124 was upregulated in sevoflurane anesthesia-induced neuroblastoma $(p<0.05)$. After miR-124 knockdown, apoptosis was significantly reduced and cell viability was enhanced in sevoflurane anesthesia-induced SMS-KAN nerve cells $(p<0.05)$. Furthermore, a significant reduction of luciferase activity was observed in 293T cells co-transfected with miR-124 mimics and EZH2-wild type (EZH2-WT) $(p<0.05)$. The mRNA and protein expression levels of EZH2 decreased in SMS-KAN nerve cells transfected with miR-124 mimics $(p<0.05)$. Overexpression of EZH2 inhibited the apoptosis of SMSKAN cells induced by sevoflurane $(p<0.05)$. Furthermore, the apoptosis of SMS-KAN cells transfected with miR-124 inhibitor were offset by transfected siEZH2.

Conclusion: The results suggest that overexpression of miR-124 suppresses cell proliferation by targeting EZH2 in SMS-KAN cells. Therefore, miR-124 represents a potential target for neuroblastoma therapy.
\end{abstract}

Keywords: Sevoflurane, Anesthesia, Neuroblastoma, Neurotoxicity, MiR-124, enhancer of zeste homolog 2 (EZH2), Apoptosis

This is an Open Access article that uses a funding model which does not charge readers or their institutions for access and distributed under the terms of the Creative Commons Attribution License (http://creativecommons.org/licenses/by/4.0) and the Budapest Open Access Initiative (http://www.budapestopenaccessinitiative.org/read), which permit unrestricted use, distribution, and reproduction in any medium, provided the original work is properly credited.

Tropical Journal of Pharmaceutical Research is indexed by Science Citation Index (SciSearch), Scopus, International Pharmaceutical Abstract, Chemical Abstracts, Embase, Index Copernicus, EBSCO, African Index Medicus, JournalSeek, Journal Citation Reports/Science Edition, Directory of Open Access Journals (DOAJ), African Journal Online, Bioline International, Open-J-Gate and Pharmacy Abstracts

\section{INTRODUCTION}

The rapid development period of human brain is from embryonic stage to 2 years after birth [1]. During this period, brain neurons grow rapidly, forming a large number of axons, dendrites and synapses. It has been reported that nerve cells are very sensitive to general anesthesia. It affects the development of the central nervous system. With the progress of medical care, more 
and more surgical operations can be used to treat infants. As a result, more and more infants are receiving general anesthesia. Sevoflurane is a commonly used general anesthetic, and is widely used in infant anesthesia. Long-term use of sevoflurane can lead to brain neuropathy, neurological dysfunction and central nervous system degeneration, affecting infants' memory and cognitive ability.

MicroRNAs (miRNAs) are a small family of noncoding RNAs that negatively regulate the expression of target genes by altering the translation or stability of the RNA. More and more evidences show that the expression of microRNA is imbalanced in human cancer, which means that microRNA plays an important role in the progress of cancer. However, sevoflurane has been reported to cause changes in the expression of many small RNAs in liver cells, and bioinformatics analysis has shown that sevoflurane treatment can induce abnormal expression of small RNAs in rat neurons[2,3]. MiR-124 is a highly conserved miRNA and highly expressed in differentiation and mature neurons.

It has been reported that increasing the expression of miR-124 can inhibit the proliferation of medulloblastoma, cervical cancer, pancreatic cancer and breast cancer [4-6]. However, the mechanism of miR-124 in nerve injury induced by anesthesia is still unclear. Enhancer of zeste homolog 2 (EZH2) is a polycomb group (PcG) protein, which is an important protein with histone methyltransferase activity. It can trimethylate the side chain of H3K27 and silence hundreds of genes, including cell differentiation and proliferation, leading to tumorigenesis. EZH2 is not expressed or low expressed in normal tissues, but highly expressed in tumors. EZH2 is highly expressed in prostate cancer, breast cancer and gastric cancer, which is closely related to disease progression and adverse prognosis [7-9].

Some reports have shown that neuronal in stem cells are modified by EZH2, and EZH2 levels are down-regulated after neuronal differentiation [10]. High expression of EZH2 is associated with poor prognosis in neuroblastoma patients [11]. Overexpression of MIR-124 has been reported to reduce the expression of $\mathrm{EZH} 2$ in hepatocellular carcinoma cells[12]. However, there are no reports in neurons about whether miR-124 causes the decrease of EZH2 expression.

This study explored if knockdown of miR-124 could have beneficial effects on sevoflurane anesthesia-induced nerve cell apoptosis by targeting $\mathrm{EZH} 2$. Overexpression of EZH2 could resist the apoptosis of nerve cells induced by sevoflurane anesthesia. The aim of this study was to reveal the potential role of miR-124 in sevoflurane anesthesia-induced neuroblastoma cells and to determine the neurotoxic mechanism of sevoflurane in SMS-KAN cells.

\section{EXPERIMENTAL}

\section{Cell culture}

Neuroblastoma SMS-KAN cell line was provided by the Korean Cell Line Bank. Cell stocks were cultured in $5 \% \mathrm{CO}_{2}$ at $37^{\circ} \mathrm{C}$ with DMEM (Grand, Island) containing $10 \%$ FBS and antibiotics (Rockville, MD). The cells were inoculated in sterile 96-well plates in a $37{ }^{\circ} \mathrm{C}$ incubator (Thermo Fisher, 3100) with $5 \% \mathrm{CO}_{2}$. To investigate the effect of sevoflurane on SMSKAN cells, $3 \%$ sevoflurane (Abbott; Chicago, IL) was added to the 96 well plates. The plates were maintained at $37^{\circ} \mathrm{C}$ for $24 \mathrm{~h}$ with $5 \% \mathrm{CO}_{2}$.

\section{Transfection of miRNA/siRNA}

The miR-124 inhibitor, control inhibitors, and small interfering RNA (siRNA) targeting EZH2 were designed, synthesized (Guangzhou, China), transfected into SMS-KAN cells (Guangzhou, China). For transfection, $1 \times 10^{5}$ cells were seeded into 96-well plates. When the cells reached $80 \%$ confluence, they were transfected with miR-124 inhibitor or control inhibitors. For EZH2 or siRNA, cells were transfected at $30-40 \%$ confluence using Lipofectamine 2000 reagent, as described previously.

\section{Quantitative real-time PCR}

Total RNA was extracted using TRIzol $B$ Reagent (CA, USA). Expressions of miR-124 and $E Z H 2$ were quantitated in rat tissues and SMSKAN cells using a real-time PCR miRNA kit. Quantitative RT-PCR reactions were performed using the ABI Prism 7500 (Applied Biosystems). The relative expression of microRNA was determined by the $\Delta \Delta \mathrm{Ct}$ value. GAPDH was used as an internal control to standardize the relative quantification of tested gene expression. The real-time qRT-PCR results were calculated by $2^{-}{ }^{-} \mathrm{Ct}$ method, in which $\Delta \Delta \mathrm{CT}=(\mathrm{Ct}$ target gene-Ct housekeeping gene) group 1- (Ct target gene-Ct housekeeping gene) group 2 . The incubation was initiated at $37^{\circ} \mathrm{C}$ for $15 \mathrm{~min}$. This was followed by $95^{\circ} \mathrm{C}$ for $30 \mathrm{sec}, 95^{\circ} \mathrm{C}$ for 5 sec, $60^{\circ} \mathrm{C}$ for $34 \mathrm{sec}$, and 40 cycles at $95^{\circ} \mathrm{C}$ for $15 \mathrm{sec}, 65^{\circ} \mathrm{C}$ for $1 \mathrm{~min}$, and $95^{\circ} \mathrm{C}$ for $15 \mathrm{sec}$. Each sample had three replicates, and the experiment was repeated three times. All primers 
used for the experiment can be found in the Table 1.

\section{MTT assay}

The cells were plated in 96-well plates at $10 \times$ $10^{4}$ per well. Then, $50 \mu \mathrm{L}$ of sevoflurane $(0,5$, 10, 20, $30 \mu \mathrm{M} / \mathrm{L})$ were added to the plates. Each group had five replicates. The blank control group used a similar volume of DMEM. The 96well plates were maintained at $37{ }^{\circ} \mathrm{C}$ for $48 \mathrm{~h}$ with $5 \% \mathrm{CO}_{2}$. Then, $20 \mu \mathrm{L}$ MTT was added to each well. After $4 \mathrm{~h}$, the supernatant was discarded, and each well added 110 $\mu$ l Formazan. The OD value at A490 $\mathrm{nm}$ of each well was detected using MK3 Enzyme Sign (Dutch Thunder Suddenly Company). The cell proliferation rate was measured according to the reference [14].

\section{Flow cytometry}

The cells were seeded on plates for $12 \mathrm{~h}$ and treated with $0.25 \%$ trypsin for $24 \mathrm{~h}$. Cells were washed three times with phosphate buffered saline (PBS). The cell suspension was evaluated for apoptosis using Annexin V-FITC (CA, USA). Apoptotic cells were detected using a FACSC alibur flow cytometer (BD Biosciences, CA).

\section{Luciferase reporter assay}

The 3'UTR of EZH2 was amplified using the Takara PCR Amplification Kit (Dalian, China). The cells were cultured in 96-well plates. Plasmids and miR-124-3p mimics were cotransfected into 293T cells. The experimental groups were as follows: control group, miR-124$3 p$, NC-inhibitor, and miR-124-3p -inhibitor. Each group was assayed in triplicate. After $24 \mathrm{~h}$, the cells reporter activities were determined using the Luciferase Reporter Assay Kit (Promega, USA).

\section{Western blot test}

Cells were washed three times with PBS and pre-cooled cell lysate was added to the cells. The solution was fully mixed, put on ice for $20 \mathrm{~min}$, and centrifuged at $12,000 \times \mathrm{g}$ for $20 \mathrm{~min}$. The protein was blocked with $5 \%$ skim milk overnight at $4{ }^{\circ} \mathrm{C}$ with antibodies after membrane transfer. After washing three times with TBS the membranes were incubated in anti-caspase-3 and mouse monoclonal anti- $\beta$-actin at a dilution of $1: 2000$ at $37^{\circ} \mathrm{C}$ for $1 \mathrm{~h}$. The protein bands were developed with ECL reagents, and images were acquired using the ChemiDoc Imaging system. Western blots were repeated at least three times.

\section{Statistical analysis}

Data are expressed as mean \pm standard deviation (SD) and were analyzed using one-way analysis of variance (ANOVA). The significance threshold was set at $p<0.05$. SPSS 17.0 was used for statistical analysis.

\section{RESULTS}

\section{Sevoflurane treatment induces apoptosis of SMS-KAN cells, upregulate miR-124 expression, and downregulates EZH2 protein expression}

The effects of sevoflurane at different concentrations on the cell viability, miR-124 expression, EZH2 expression and apoptosis of SMS-KAN cells were evaluated by MTT assay, flow cytometry, qRT-PCR and WB testing. As shown in Figures $1 \mathrm{~A}$ and $1 \mathrm{C}$, compared with control, the cell viability and EZH2 protein expression significantly decreased as sevoflurane concentration increased $(p<0.05)$. However, with the increase of sevoflurane concentration, the expression of miR-124 and apoptosis of SMS-KAN cells increased significantly $(p<0.05)$, as shown in Figure $1 \mathrm{~B}$ and $D$. These results demonstrate that sevoflurane treatment induced apoptosis in SMSKAN cells, directly increased the expression of miR-124 and decreased the expression of EZH2 protein.

Knockdown of miR-124 suppresses the apoptosis of SMS-KAN cells induced by sevoflurane

In order to study the role of miR-124 in SMSKAN cells, inhibitor knockout of miR-124 was transfected into sevoflurane-treated SMS-KAN cells. The expression of miR-124 was confirmed by qrt-pcr.

Table 1: Sequence of primer design

\begin{tabular}{lllc}
\hline Gene & Primer forward (5'-3') & Primer reverse (5'-3') & Size (bp) \\
\hline miR-124 & ATCCAGTGCGTGTCGTG CTC T & TGCTTAAGGCACGCGGT TAG AA & 153 \\
EZH2 & GACGGCTTCCCAATAACAG & ATTGAGGCTTCAGCACCAC AC & 149 \\
\hline
\end{tabular}




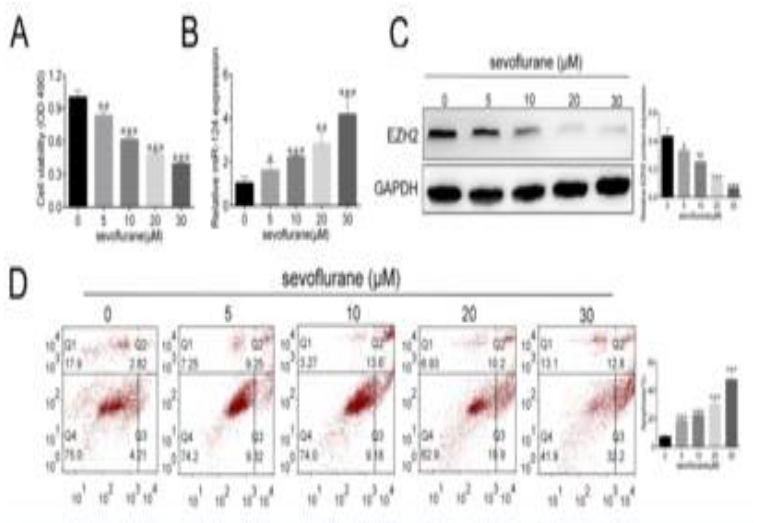

Figure 1: Sevoflurane induced apoptosis of sms-kan cells, up-regulated the expression of miR-124 and down-regulated the expression of EZH2 protein. (A) The cell viability of SMS-KAN cells was determined using the MTT assay. (B) qRT-PCR analysis of miR124 expression in SMS-KAN cells. (C) EZH2 protein expression was measured using western blot quantification. (D) Apoptosis was determined using flow cytometry; ${ }^{*} p<0.05,{ }^{* *} p<0.001$ and ${ }^{* * *} p<$ 0.0001 versus NC. All experiments were carried out in replicate

The results showed that miR-124 knockdown in SMS-KAN cells was successful $(p<0.05)$ (Figure 2 A). miR-124 knockdown in SMS-KAN cells was successful. As shown in Figure 2 B, transfection withmiR-124 inhibitor effectively increased the expression of $\mathrm{Bcl}-2$ protein, as determined by western blotting. The expressions of Bax and cleaved caspase-3 protein decreased. Treatment with the miR-124 inhibitor reduced SMS-KAN cell apoptosis (Figure $2 \mathrm{C}$ ). These results show that the miR-124 inhibitor has repressive effects on the apoptosis of SMSKAN cells induced by sevoflurane.

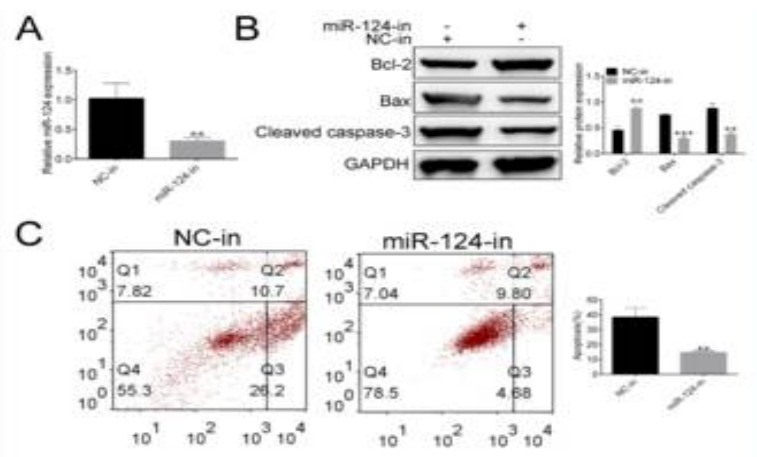

Figure 2: Knockdown of miR-124 attenuated the apoptosis induced by sevoflurane treatment. (A) miR124 expression levels were determined using qRTPCR. (B)Bcl-2, Bax, and cleaved caspase-3 protein levels were detected using western blotting. (C) Apoptosis was detected using flow cytometry; ${ }^{*} p<$ $0.05,{ }^{* *} p<0.001$ and ${ }^{* * *} p<0.0001$ versus NC. All experiments were performed in replicate
EZH2 is a direct target of miR-124 in SMSKAN cells

TargetScan Release (http://www.targetscan.org/) predicted a binding site for EZH2 with miR-124. In this study, 293T cells transfected with miR-124 were analyzed by using EZH2-3'UTR-wild or EZH2-3'UTR-mut (Fig. 3A). The luciferase activity of the EZH2-3'UTR-wild plasmid decreased following treatment with miR-124. The suppression effect of the miR-124 was not observed with the EZH2-3'UTR-mut (Figure 3 B). Western blot testing found that the expression of EZH2 protein was decreased after treatment with miR-124 in 293T cells (Figure $3 \mathrm{C}$ ). This may be due to the inhibition of gene expression by miR124 binding to 3' UTR of EZH2 mRNA.

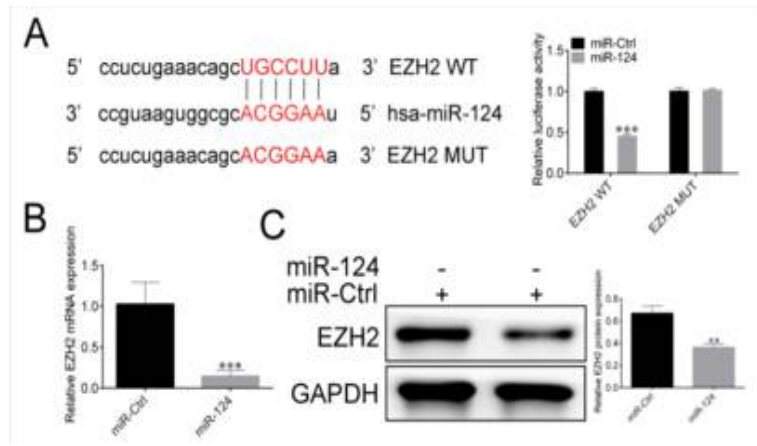

Figure 3: MiR-124 regulates EZH2 expression. (A)TargetScan predicted an miR-124 target sequence. (B) The mRNA levels of EZH2 were detected using RT-PCR. (C)EZH2 protein levels were detected using western blotting; ${ }^{*} p<0.05,{ }^{* *} p<0.001$ and ${ }^{* * *} p<$ 0.0001 versus NC. All experiments were conducted in replicate

\section{Overexpression of EZH2 inhibits apoptosis of SMS-KAN cells induced by sevoflurane}

We transfected si-EZH2 into SMS-KAN cells to determine if EZH2 plays a role in SMS-KAN cells. The transfection effectively increased $\mathrm{EZH} 2$ at the mRNA and protein expression levels, as tested by qRT-PCR and western blotting, respectively $(p<0.05)$ (Figure $4 \mathrm{~A}$ and $B)$. Flow cytometry analysis found that overexpression of EZH2 can resist apoptosis in sevoflurane-treated SMS-KAN cells $(p<0.05$, Figure $4 \mathrm{C}$ ). As shown in Figure $4 \mathrm{D}$, overexpression of EZH2effectively increased the expression of $\mathrm{Bcl}-2$ protein, whereas the expression of Bax and cleaved caspase- 3 protein decreased $(p<0.05)$. Therefore, the overexpression of EZH2 inhibits the apoptosis of sms-kan cells induced by sevoflurane. 


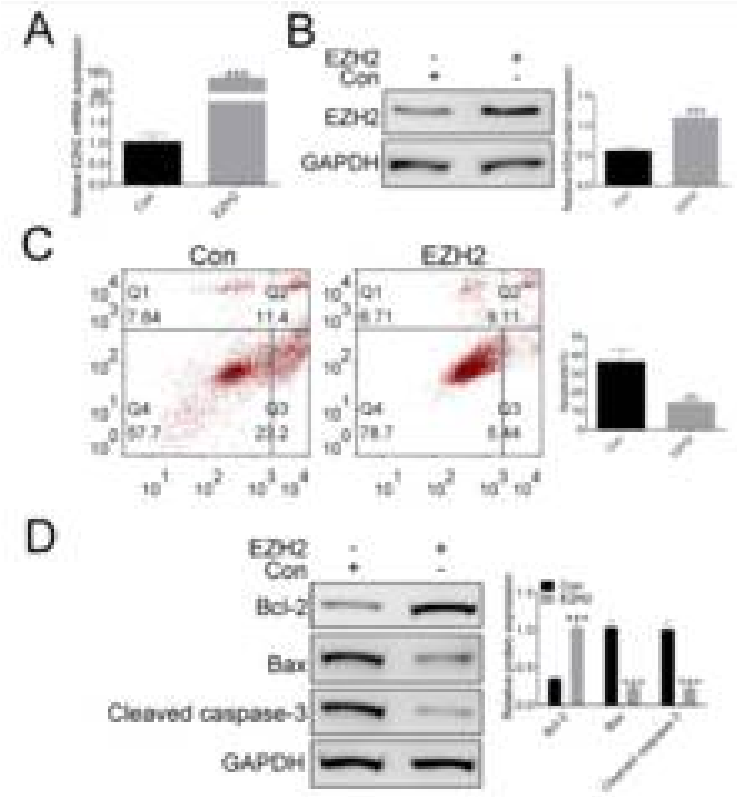

Figure 4: Overexpression of EZH2 inhibits the apoptosis of SMS-KAN cells induced by sevoflurane. (A) EZH2 mRNA expression was determined using qRT-PCR. (B) EZH2 protein expression was determined using qRT-PCR. (C) Apoptosis was determined using flow cytometry. (D)Bcl-2, Bax, and cleaved caspase-3 protein levels were determined using western blotting; ${ }^{*} p<0.05,{ }^{* *} p<0.001$ and ${ }^{* * *} p$ $<0.0001$ versus NC. All experiments were carried out in replicate

\section{Mir-124 regulates sevoflurane-induced SMS- KAN cells apoptosis by targeting EZH2}

We co-transfected miR124 inhibitor and siEZH2 in SMS-KAN cells to explore if the function of miR-124 was associated with EZH2 in SMS-KAN cells treated with sevoflurane. Apoptosis were confirmed by flow cytometry. The results showed that compared with NC-in + siRNA, there was no significant difference in apoptosis in NC-in + siEZH2, but the apoptosis in the miR-124-in + siRNA cells was significantly reduced $(p<0.05$, Figure $5 \mathrm{~A}$ ). Western blots showed that compared with NC-in + siRNA, the EZH2 and $\mathrm{Bcl}-2$ expressions of miR-124-in + siRNA significantly increased. However, the Bax and cleaved caspase-3 expression significantly decreased $(p<0.05$, Figure $5 \mathrm{~B})$. These data suggest that MIR-124 targeting EZH2 regulates sevoflurane-induced apoptosis of SMS-KAN cells.

\section{DISCUSSION}

Anesthetic exposure to sevoflurane induced cerebral neuronal apoptosis and cognitive dysfunction, especially in infancy [15]. It has been reported that neurons exposed to $3 \%$ sevoflurane for more than 6 hours can induce nearly $40 \%$ cell apoptosis [16]. Liang et al showed that sevoflurane induces cell proliferation and apoptosis of A549 cells [17]. This study found that sevoflurane reduced cell viability, inhibited EZH2 protein expression, and induced apoptosis of sms-kan cells, similar to previous reports.

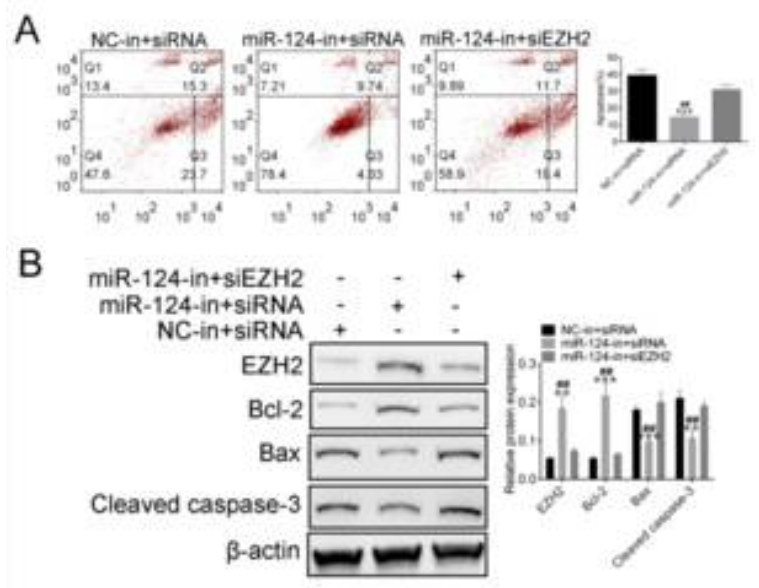

Figure 5: MiR-124 targets $\mathrm{EZH} 2$ to modulate the apoptosis of SMS-KAN cells induced by sevoflurane. (A) The apoptosis in cells transfected with NC-in + siRNA, miR-124-in + siRNA, and miR-124-in + siEZH2. (B) The protein expression of $\mathrm{EZH} 2, \mathrm{Bcl}-2$, Bax, and cleaved caspase- $3 ;{ }^{*} p<0.05,{ }^{* *} p<0.001$ and ${ }^{* * *} p<0.0001$ versus NC. All experiments were replicated at least three times. miR-124-in: miR-124 inhibitor

Recent studies have shown that mutations related to miRNAs and changes in the expression level of miRNAs have important effects on post-transcriptional regulation of target genes, changes in the expression level of target proteins and drug sensitivity of cancer cells [18]. Abnormal expression of miR-124 may cause various human malignant tumors. In oral squamous cell carcinomas, the expression of microRNA-124 is low, which induces proliferation, differentiation, cell cycle arrest and apoptosis of cancer cells by targeting EZH2 [19].

The down-regulation of microRNA-124 expression in gastric cancer cells is closely related to the degree of differentiation, clinical stage and lymph node metastasis of gastric cancer [20]. At present, there are relatively few studies on the role of miRNAs in nerve injury. In this study, miR-124 and EZH2 may have a targeting relationship by bioinformatics and play an important regulatory role in the development of sevoflurane-induced SMS-KAN cells apoptosis. In this study, we found that miR-124 expression was up-regulated in SMS-KAN cells treated with sevoflurane. The results also indicate that miR-124 and EZH2 have a targeted 
regulatory relationship and play an important regulatory role in the occurrence and development of neuroblastoma. In addition, we constructed recombinant eukaryotic vectors to transfect SMS-KAN cells and performed MTT analysis, which confirmed that overexpression of miR-124 could increase sevoflurane-induced neuronal apoptosis.

$\mathrm{EZH} 2$ is a homologous human gene of zeste gene enhancer in Drosophila melanogaster. It is an important member of PcG (Polycomb Group) gene family [21] [22]. Abnormal expression of EZH2 may induce the occurrence of multiple tumors. EZH2 promotes cell proliferation and proliferation by inhibiting related target gene [23]. In glioblastoma, EZH2 promotes the proliferation, migration and angiogenesis of tumor cells. The lymph node metastasis rate increases with the increase of EZH2 expression. [24]. Using luciferase reporter assays, it was found that fluorescence activity was significantly lower after transfection of EZH2 and miR-124-WT in 293T cells. qRT-PCR and western blot testing also verified that miR-124 could inhibit the mRNA and protein expression levels of EZH2. This suggests that overexpression of miR-124 could inhibit the proliferation activity of SMS-KAN nerve cells by targeting the expression of EZH2.

\section{CONCLUSION}

The findings of this study revealed that miR-124 expression is upregulated in SMS-KAN cells in response to sevoflurane anesthesia. Knock out of miR-124 inhibits the apoptosis of neurons induced by sevoflurane. Overexpression of EZH2 inhibits apoptosis, while MIR-124 targeting of EZH2 regulates sevoflurane-induced neuronal apoptosis. Thus, the results indicate that miR124 may be a diagnostic marker and potential therapeutic target of anesthesia-induced neuroblastoma.

\section{DECLARATIONS}

\section{Conflict of Interest}

No conflict of interest associated with this work.

\section{Contribution of Authors}

We declare that this work was done by the researchers listed in this article. All liabilities related with the content of this article will be borne by the authors. Lingyun Wei designed all the experiments and revised the paper. Jiansheng Fang and Yiqi Fang formed the experiments, Chengnv Li wrote the manuscript.

\section{REFERENCES}

1. Ankarberg E. Neurotoxic effects of nicotine during neonatal brain development: critical period and adult susceptibility. Acta Univer Upsal. 2003; 1: 7989-7994.

2. Ishikawa M, Tanaka S, Arai M, Genda Y, Sakamoto A. Differences in microRNA changes of healthy rat liver between sevoflurane and propofol anesthesia. Anesthesiology. 2012; 117(6): 1245-1252.

3. Ye J, Zhang Z, Wang $Y$, Chen $C, X u X, Y u H$, Peng $M$. Altered hippocampal microRNA expression profiles in neonatal rats caused by sevoflurane anesthesia: MicroRNA profiling and bioinformatics target analysis. Exp Ther Med. 2016; 12(3): 1299-1310.

4. Wilting $S M$, van Boerdonk RA, Henken FE, Meijer CJ, Diosdado B, Meijer GA, Le SC, Agami R, Snijders PJ, Steenbergen RDJCG et al. Methylation-mediated silencing and tumour suppressive function of hsa-miR124 in cervical cancer. Mol Cancer. 2010; 203(1): 167177.

5. Wang $P$, Chen L, Zhang J, Chen H, Fan J, Wang K, Luo J, Chen Z, Meng Z, Liu LJO. Methylation-mediated silencing of the miR-124 genes facilitates pancreatic cancer progression and metastasis by targeting Rac1.Oncogene. 2014; 33(4): 514-524.

6. Yong-Jun L, Qiu-Yu W, Ci-Xiang Z, Qian-Qian Y, Ming $H$, Xiao-Ting $Y$, Dan-Xia C, Guo-Qiang $C$, Jian-Rong $H$, Qian ZJC. MiR-124 targets Slug to regulate epithelialmesenchymal transition and metastasis of breast cancer. Carcinogenesis. 2013; 34(3): 713-722.

7. Sooryanarayana V, Dhanasekaran SM, Ming Z, Barrette TR, Chandan KS, Sanda MG, Debashis G, Pienta KJ, Sewalt RGAB, Otte AP. The polycomb group protein $E Z H 2$ is involved in progression of prostate cancer. Nature. 2002; 419(6907): 624-629.

8. Kleer CG, Qi C, Sooryanarayana V, Ronglai S, Ichiro $O$, Tomlins SA, Debashis G, Sewalt RGAB, Otte AP, Hayes DF. Proceedings of the National Academy of Sciences of the United States of America. EZH2 is a marker of aggressive breast cancer and promotes neoplastic transformation of breast epithelial cells. Proc Natl Acad Sci U S A. 2003; 100(20): 11606-11611.

9. Cai GH, Wang K, Miao Q, Peng YS, Chen X. Expression of polycomb protein EZH2 in multi-stage tissues of gastric carcinogenesis. J Dig Dis .2010; 11(2): 88-93.

10. Ezhkova E, Pasolli HA, Parker JS, Stokes N, Su I, Hannon G, Tarakhovsky A, Fuchs E. Ezh2 Orchestrates Gene Expression for the Stepwise Differentiation of Tissue-Specific Stem Cells. Cell. 2009; 136(6): 11221135.

11. $Z L, H T, A N S, N A, M H, S S, Y S, K C, K M, J A . E Z H 2$ regulates neuroblastoma cell differentiation via NTRK1 promoter epigenetic modifications. Oncogene. 2018; 37(20): 2714.

12. Zheng F, Liao YJ, Cai MY, Liu YH, Liu TH, Chen SP, Bian $X W$, Guan $X Y$, Lin MC, Zeng $Y X$. The putative tumour suppressor microRNA-124 modulates hepatocellular carcinoma cell aggressiveness by 
repressing ROCK2 and EZH2. Gut. 2012; 61(2): 278289.

13. Wang $Z$, Yin $H$, Zhang $Y$, Feng $Y$, Yan Z, Jiang $X$, Bukhari I, lqbal F, Cooke HJ, Shi Q. miR-214-mediated downregulation of RNF8 induces chromosomal instability in ovarian cancer cells. Cell Cycle. 2014; 13(22): 3519-3528.

14. Chen XY, Li J, Cheng WM, Jiang H, Xie XF, Hu R. Effect of total flavonoids of Chrysanthemum indicum on the apoptosis of synoviocytes in joint of adjuvant arthritis rats. Am J Chin Med. 2008; 36(04): 695-704.

15. Jevtovictodorovic $V$, Hartman RE, Izumi $Y$, Benshoff $N D$, Dikranian K, Zorumski CF, Olney JW, Wozniak DF. Early Exposure to Common Anesthetic Agents Causes Widespread Neurodegeneration in the Developing Rat Brain and Persistent Learning Deficits. J Soc Neurosci. 2003; 23(3): 876.

16. Wang WY, Yang R, Hu SF, Wang H, Ma ZW, Lu Y. Nstearoyl- I -tyrosine ameliorates sevoflurane induced neuroapoptosis via MEK/ERK1/2 MAPK signaling pathway in the developing brain. Neurosci Lett. 2013; 541(1): 167-172.

17. Liang $H$, Gu MN, Yang CX, Wang HB, Wen XJ, Zhou QL. Sevoflurane inhibits proliferation, induces apoptosis, and blocks cell cycle progression of lung carcinoma cells. Asian Pac J Cancer Prev. 2011; 12(12): 3415-3420.

18. Mostert B, Sieuwerts AM, Galen AMV, Vries JB, Kraan J, Lalmahomed Z, Verhoef C, ljzermans JNM, Weerd VD, Spoel PVD. Abstract 3409: Generation of mRNA and miRNA gene expression profiles in circulating tumor cells of metastatic colorectal cancer patients. Cancer Res. 2012; 72(8 Supplement): 3409-3409.
19. Hunt $S$, Jones AV, Hinsley EE, Whawell $S A$, Lambert DW. MicroRNA-124 suppresses oral squamous cell carcinoma motility by targeting ITGB1. Febs Lett. 2011; 585(1): 187-192.

20. Li KKW, Pang CS, Ching KK, Wong CK, Kong $X$, Wang $Y$, Zhou L, Chen Z, Ng HK. miR-124 is frequently downregulated in medulloblastoma and is a negative regulator of SLC16A1. Hum Pathol. 2009; 40(9): 12341243.

21. Ahani $N$, Shirkoohi $R$, Rokouei $M$, Eskandani $M A$, Nikravesh $A$. Overexpression of enhancer of zeste human homolog 2 (EZH2) gene in human cytomegalovirus positive glioblastoma multiforme tissues. Med Oncol. 2014; 31(11): 1-5.

22. Breuer RHJ, Snijders PJF, Smit EF, Sutedja TG, Sewalt RGAB, Otte AP, Kemenade FJV, Postmus PE, Meijer CJLM, Raaphorst FM. Increased Expression of the EZH2 Polycomb Group Gene in BMI-1-Positive Neoplastic Cells during Bronchial Carcinogenesis. Neoplasia. 2004; 6(6): 736-743.

23. EV K, AS R, AT G, NP A, CG T, JXZ, RD C, AD B, HW C. ANCCA/ATAD2 overexpression identifies breast cancer patients with poor prognosis, acting to drive proliferation and survival of triple-negative cells through control of B-Myb and EZH2. Cancer Res. 2010; 70(22): 9402.

24. Suvã ML, Riggi N, Janiszewska M, Radovanovic I, Provero P, Stehle JC, Baumer K, Le BM, Marino D, Cironi L. EZH2 is essential for glioblastoma cancer stem cell maintenance. Cancer Res. 2009; 69(24): 92119218. 